\title{
Relación entre gramática y escritura en el currículo de Lengua Portuguesa
}

\author{
Madalena Teixeira \\ Centro de Estudos Anglísticos (CEAUL), Universidade de Lisboa
}

Artículo recibido 5 de junio de 2012

\begin{abstract}
En este trabajo se analiza la relación entre los dominios de la gramática y de la escritura en el diseño curricular portugués, con vista a la comprensión de los resultados de los exámenes que se observan en las pruebas oficiales. Estas pruebas arrojan resultados muy bajos. Para realizar este análisis nos basamos en los principios definidos para la enseñanza de la gramática de dos documentos oficiales: los Programas del área curricular de Portugués (1er y $2^{\circ}$ ciclos de la Enseñanza Básica) y la asignatura de Portugués (3er ciclo de la enseñanza Básica) (2009) y las Metas Curriculares de Portugués para la Enseñanza Básica (2012). Estos documentos son normativos y funcionan como referentes para el profesorado en relación tanto a los diseños curriculares de aula como al desarrollo de la práctica docente y a la evaluación interna de los aprendizajes y de los libros de texto.

A partir de aquí nos planteamos los siguientes objetivos: (1) conocer el impacto de la relación entre los dominios de la gramática y de la escritura, en el contexto del sistema nacional de evaluación; (2) describir el marco teórico y los mecanismos didácticos de los Programas (2009) y las Metas (2012); y (3) proponer actividades didácticas para la enseñanza de la gramática en articulación con la escritura. Como conclusión, señalamos como una prioridad la articulación entre los Programas del área curricular de lengua y la inversión en la formación continuada de los docentes.
\end{abstract}

\section{Introducción}

Este trabajo ${ }^{\mathrm{i}}$ tiene como principal objetivo reflexionar sobre la relación entre gramática y escritura en los documentos oficiales en Portugal. Los resultados obtenidos por los alumnos en los exámenes nacionales evidencian fragilidades que han culminado en los "peores resultados" en la prueba de Conocimiento de la Lengua y de la Escritura, de acuerdo con diversos informes oficiales (GAVE, 2010, 2011 y 2012).

En este texto no tenemos la intención de evaluar el diseño curricular base y los documentos que lo configuran. Esta intención implicaría la diferenciación de dos importantes dimensiones: la formativa (desarrollo del proceso) y la sumativa (evaluación de su resultado) (Scriven, 1967). Sí podemos explorar los documentos que rigen la práctica docente. El portugués es lengua de escolarización y de conocimiento en el sistema educativo portugués (Sá, 2012) y por ello elemento fundamental en todo el proceso de enseñanza y aprendizaje. Además, es esencial 
comprender cómo estos documentos articulan la relación entre gramática y escritura. Ello puede permitirnos dar una eventual explicación a los resultados observados en los exámenes nacionales.

Así, en un primer momento intentaremos identificar los puntos en los cuales sea evidente en los documentos oficiales la interrelación entre gramática y escritura. Mostraremos el marco teórico de los Programas (Programas, 2009) y de las Metas (Metas, 2012) ${ }^{\mathrm{ii}}$, dando particular énfasis a la competencia "Conocimiento Explícito de la Lengua" y al dominio "Gramática", así como a Escritura. Exploraremos las similitudes y diferencias entre los dos documentos y las implicaciones en términos de resultados de la evaluación (éxito vs. fracaso del alumnado). Seguidamente nos detendremos en la relación entre gramática y escritura en ambos documentos, con el objetivo de comprender sus concepciones subyacentes. Con este procedimiento intentamos averiguar se los resultados obtenidos en los Exámenes pueden ser consecuencia de la incapacidad de estos documentos a la hora de orientar con claridad al profesorado.

Presentaremos asimismo dos muestras de actividades didácticas para el desarrollo de la competencia específica Conocimiento Explícito de la Lengua. Estas actividades se basan muestran las implicaciones del conocimiento gramatical en el desarrollo de la expresión escrita. Por último, presentamos unas consideraciones finales, que sugieren la necesidad de articulación entre textos reguladores de la práctica docente (Programa, 2009, y Metas, 2012) y una apuesta por la formación continua del profesorado.

\section{Los Informes de los Exámenes Nacionales}

Los Exámenes Nacionales, en Portugal, implantados con la intención de averiguar los conocimientos del alumnado en diferentes áreas, han sido objeto de atención legislativa en los últimos años culminando actualmente en su obligatoriedad para todos los ciclos de la enseñanza básica y secundaria.

Debemos considerar que no siempre incluyen información de todos los niveles de enseñanza. Por ejemplo, en 2009 los alumnos de los cuatro primeros cursos de primaria (el llamado Primer Ciclo) no realizaron esta prueba, que sí realizaron en el curso 2012/2013 (bajo la denominación Prueba Final de Ciclo). Así, en el informe del 2009, puede leerse que existe un desconocimiento del “...funcionamiento de la 
lengua en los niveles de conocimientos menos elementares, bien en lo que respeta al dominio correcto de la lengua portuguesa, bien en lo relativo al dominio del metalenguaje..." (GAVE, 2010, pp. 6-11), quedando las dificultades detectadas, en la Escritura, confinadas al texto expositivo-argumentativo.

Los dados del informe de 2010 muestran que solamente el 24,6\% de los alumnos supo contestar correctamente el apartado llamado Grupo II, destinado a evaluar el conocimiento lingüístico (GAVE, 2010). Este informe apunta como posible explicación que los contenidos se habían trabajado sólo aquel curso. Ello indicaría la necesidad de trabajar las nociones oracionales en períodos más largos de tiempo. En lo que concierne a la escritura, se evaluaron los siguientes aspectos: (a) tema y tipología, (b) coherencia y pertinencia de la información, (c) estructura y cohesión, (d) morfología y sintaxis, (e) repertorio léxico; y (f) ortografía. El alumnado tuvo dificultades en lo que se reporta a los puntos (c) y (f), lo que nos lleva a pensar en el importante rol que pueden jugar los conocimientos gramaticales.

Los informes de 2011 y 2012 apuntan en la misma dirección (GAVE, 2011 y 2012). Muestran también un menor nivel de respuestas correctas en el Grupo II y de nuevo un bajo nivel de acierto en el ámbito (c) de estructura y cohesión. Muestran asimismo problemas en el ámbito (d) de "morfología y sintaxis". Las propuestas de intervención didáctica indicadas en ambos informes son similares. Tal como se indica en el informe de 2012, es esencial trabajar la

sistematización del conocimiento explícito en lo relativo a los mecanismos de cohesión textual, morfología verbal y arquitectura de la frase. Importa igualmente reforzar que el entrenamiento de ampliación del capital lexical y adecuación del vocabulario, bien como promover prácticas de revisión textual que incluyan el plan ortográfico (...) los resultados confirman la necesidad de refuerzo de la enseñanza de la gramática. (GAVE, 2012, pp.13, 75)

En relación al 2013 el informe GAVE (2013) corrobora los resultados anteriores y revela aprendizajes todavía insuficientes. En este documento, que destaca la necesidad de disminuir los porcentajes de suspensos al final del primer ciclo, puede leerse que los peores resultados del alumnado se observan en los ámbitos de escritura y gramática (en lo que se conoce como Conocimiento Explícito de la Lengua, CEL) (GAVE, 2013).

El informe destaca los siguientes problemas en relación al conocimiento explícito de la lengua. En el plano morfológico, dificultades para reconocer la flexión 
en género y número en contexto de ítems de asociación y de correspondencia (sólo un $35,2 \%$ y un $35,9 \%$ de éxito respectivamente). En el plano de la representación gráfica y ortográfica, dificultades en el uso de acentos gráficos (agudos, graves y circunflejos) (con sólo el 25,5\% de éxito). Y en el plano sintáctico, problemas en la sustitución de un grupo nominal (en contexto de oración y sin recurso al metalenguaje) en tareas con ítems de elección múltiple (con el 45,8\% de éxito) (GAVE, 2013).

En relación a la Escritura, sólo un 39,6\% del alumnado logró escribir un texto coherente y sólo un 37,8\% utilizó niveles de vocabulario adecuados. Los alumnos mostraron especiales problemas en la corrección ortográfica (GAVE, 2013), con sólo un $24,7 \%$ de textos correctos.

Un análisis de estos informes permite destacar cuatro aspectos. En primer lugar, los resultados obtenidos en el dominio de la Escritura parecen derivar de las debilidades relativas al conocimiento explícito del sistema de la lengua, pues los indicadores apuntan a problemas relacionados con los mecanismos de estructura textual, coherencia, cohesión, sintaxis, morfología, puntuación y acentuación. Ha sido destacado que probablemente estos problemas afecten también los niveles de éxito en los dominios de Lectura y de Oralidad y en definitiva de la competencia comunicativa (Travaglia, 2007).

En segundo lugar, en el proceso de elaboración de los exámenes no fueron incluidos los contenidos de gramática indicados en las Metas (2012), a pesar de que estos contenidos se presentaban como el punto de referencia para la enseñanza y el aprendizaje y para la evaluación interna y externa (p. 4). En tercer lugar, finalmente, la nomenclatura utilizada en los Exámenes Nacionales es conforme a los Programas (Programa, 2009) pero en ocasiones no coincide con la nomenclatura de las Metas (2012). Mientras que el primer documento usa la designación Conocimiento Explícito de la Lengua, en el segundo usa el término Gramática; no obstante a priori las Metas (2012) respetan globalmente "los dominios existentes [en los Programas, 2009)] (Oralidad, Lectura, Escritura y Conocimiento Explícito de la Lengua, ahora designado Gramática)...” (p.6). Consideramos pertinente por lo tanto explorar las semejanzas y diferencias entre los dos documentos. 


\section{Dos documentos oficiales: Programas (2009) y Metas (2012)}

\section{Marco teórico}

Tanto los Programas (2009), como las Metas (2012) presentan como objetivo fundamental contribuir a la mejora de los aprendizajes del alumnado, basado en determinados principios que se fundamentan en la valoración de la progresión en el aprendizaje con niveles crecientes de complejidad. Ello puede entreverse en afirmaciones como "valoración del principio de la progresión" (Programa, 2009, p. 9) o "conocimientos a adquirir y las capacidades que se quieren ver desarrollar, respetando el orden de progresión en su adquisición." (Metas, 2012, p.1).

En los dos documentos se constata una revalorización del rol de la gramática. Ambos defienden que el trabajo sobre gramática se inicie ya en los primeros cursos de enseñanza básica $\left(1^{\circ}\right.$ y $\left.2^{\circ}\right)$ mientras que en las anteriores disposiciones curriculares (de 1991) el inicio de la enseñanza gramatical se sitúa en $3^{\circ}$. Por otro lado, en los Programas (2009) se plantea la necesidad de crear una cultura literaria compartida y se propone un corpus textual (ver paginas 64, 104, 159-163) de referencias culturales nacionales y extranjeras. El documento Metas (2012) va más allá, creando un dominio específico, la Educación Literaria: fruto de la política de enseñanza que transmite valores y costumbres, presenta una base común de obras literarias. La necesidad de atender a este ámbito educativo se planteó por primera vez en la Conferencia Internacional del Portugués, en la cual se hicieron recomendaciones para una nueva interdisciplinaridad y la integración de los textos literarios a la enseñanza de la lengua por su singularidad estilística.

Los Programas (2009) presentan una matriz común para los tres ciclos de enseñanza básica, mientras que las Metas (2012) proponen una matriz común sólo para el primer y segundo ciclo, distinto del tercero. Se argumenta que ello responde a la mayor dificultad que entrañan los contenidos del tercer ciclo. Los Programas (2009) están organizados por competencias, comunes en los tres ciclos (comprensión oral, expresión oral, lectura, escritura y conocimiento explícito de la lengua); es remarcable el hecho de que lectura y escritura no se presentan conjuntamente en ninguno de los tres ciclos de la enseñanza básica. Por otro lado, las Metas (2012) están organizadas por dominios. Presentan cuatro dominios para el primer y segundo ciclo (oralidad, lectura y escritura, educación literaria y gramática) y cinco para el tercero (oralidad, lectura, escritura, educación literaria y gramática). 
Otra diferencia, importante, afecta al grado de maniobra que se reserva al docente. Mientras que los Programas (2009) definen los contenidos por ciclo, las Metas (2012) los definen por curso. Consideramos que ello tiene consecuencias sobre el margen de decisión reservado al docente a la hora de seleccionar los contenidos que se impartirán en cada curso. Finalmente, mientras que en los Programas (2009) para cada competencia se indican competencias específicas (así como los descriptores de nivel y los respectivos contenidos), en el caso de las Metas (2012) en cada dominio se indican los objetivos y los descriptores de nivel.

Las Metas (2012) se presentan como un documento que orienta sobre cómo usar los Programas (2009), pues indican cuáles son los contenidos prioritarios que el profesorado debería impartir en clase. Una cuestión que surge es cómo trabajar a la par con los dos documentos. Hemos elegido como ejemplo la Gramática en los primeros cursos. Observemos los Cuadros 1 y 2 siguientes, sobre la gramática en los dos documentos oficiales (Programa, 2009, y Metas, 2012) en primer y segundo curso, respectivamente.

\begin{tabular}{|c|c|c|}
\hline $\begin{array}{c}\text { Metas Curriculares } \\
\text { Domínio da Gramática- } 1^{\circ} \text { ano }\end{array}$ & $\begin{array}{r}\text { Programa } \\
\text { Competência do }\end{array}$ & $\begin{array}{l}\text { Português } \\
\text { EL - 10 e 20 anos }\end{array}$ \\
\hline $\begin{array}{l}\text { Objetivo: Descobrir regularidades } \\
\text { no funcionamento da lingua. } \\
\text { - Descritores de Desempenho: } \\
\text { Formar femininos e masculinos de } \\
\text { nomes e adjetivos de flexão regular } \\
\text { (de indice temático - ou -a). } \\
\text { Formar singulares e plurais de nomes } \\
\text { e adjetivos que seguem a regra geral } \\
\text { (acrescentar - sao singular), } \\
\text { incluindo os que terminam em -m e } \\
\text { fazem o plural em -ns (fim, bom, } \\
\text { etc.). } \\
\text { - Objetivo: Compreender formas de } \\
\text { organização do léxico. } \\
\text {-Descritores de Desempenho: } \\
\text { A partir de atividades de oralidade, } \\
\text { verificar que há palavras que têm } \\
\text { significado semelhante e outras que } \\
\text { têm significado oposto. }\end{array}$ & $\begin{array}{l}\text { Descritores de desempenho: } \\
\text { Manipular palavras e } \\
\text { constituintes de palavras e } \\
\text { observar os efeitos produzidos } \\
\text { (1) } \\
\text { - Formar femininos, } \\
\text { masculinos;singulares e plurais; } \\
\text { Produzir novas palavras a partir } \\
\text { de sufixos e prefixos; }\end{array}$ & $\begin{array}{l}\text { Conteúdos } \\
\text { - (1) Flexão nominal, adjetctival - } \\
\text { número (singular, plural); género } \\
\text { (masculino, feminino) } \\
\text { - Flexão pronominal- (singular, } \\
\text { plural); género } \\
\text { (masculino,feminino), pessoa } \\
\left(1 .{ }^{2}, 2.3 .{ }^{2}{ }^{2}\right)\end{array}$ \\
\hline
\end{tabular}


Cuadro 2. Segundo curso

\begin{tabular}{|c|c|c|}
\hline $\begin{array}{c}\text { Metas Curriculares } \\
\text { Domínio da Gramática- } 2^{\circ} \text { ano }\end{array}$ & \multicolumn{2}{|c|}{$\begin{array}{l}\text { Programa de Português } \\
\text { Competência do CEL - 19 e 2o anos }\end{array}$} \\
\hline $\begin{array}{l}\text { Objetivo: Explicitar regularidades } \\
\text { no funcionamento da lingua. } \\
\text { - Descritores de Desempenho: } \\
\text { 1. Identificar nomes. } \\
\text { 2. Identificar o determinante artigo } \\
\text { (definido e indefinido). } \\
\text { 3. Identificar verbos. } \\
\text { 4. Identificar adjeivos. } \\
\text { - objetivo: Compreender formas de } \\
\text { organização do léxico. } \\
\text {-Descritores de Desempenho: } \\
\text { A partir de atividades de oralidade e de } \\
\text { leitura, verificar que há palavras que } \\
\text { têm significado semelhante e outras } \\
\text { que têm significado oposto. }\end{array}$ & $\begin{array}{l}\text { Descritores de desempenho: } \\
\text { - Manipular palavras e constituintes } \\
\text { de palavras e observar os efeitos } \\
\text { produzidos (1) } \\
\text { - } \quad \text { Formar femininos, } \\
\text { masculinos;singulares e plurais; } \\
\text { - } \quad \text { Produzir novas palavras a partir de } \\
\text { sufixos e prefixos; } \\
\text { Comparar dados e descobrir } \\
\text { regularidades (1) } \\
\text { Explicitar: } \\
\text { Distinguir nomes, verbos e } \\
\text { adjetivos. }\end{array}$ & $\begin{array}{l}\text { Conteúdos } \\
\text { - (1) Flexão nominal, adjetctival - } \\
\text { número (singular, plural); género } \\
\text { (masculino, feminino) } \\
\text { - Flexão pronominal- (singular, } \\
\text { plural); género } \\
\text { (masculino,feminino), pessoa } \\
\left(1 \text { a }^{2} 2^{2} 3^{a}{ }^{a} \text { ) }\right. \\
\text { - Sinónimos, antónimos. } \\
\text { - Nome-próprio, comum (coletivo) } \\
\text { - Adjetivo } \\
\text { - Verbo }\end{array}$ \\
\hline
\end{tabular}

Los Cuadros 1 y 2 presentan los contenidos de los Programas (2009) (sobre la competencia Conocimiento Explícito de la Lengua) y las Metas (2012) (sobre los contenidos del dominio de la Gramática). En una primera lectura de los Programas (2009) se detectan contenidos específicos: vocales orales y nasales, consonantes, diptongos, sílabas, dígrafos, orden alfabético, verbos, tiempos verbales (presente, futuro, pretérito perfecto), frase y no frase, sujeto, predicado, grupo nominal y grupo verbal, familia de palabras, acentuación (acento agudo, grave y circunflejo), principios de cortesía, formas de tratamiento, signos de puntuación (punto, punto de interrogación, punto de exclamación, reticencias, coma, dos puntos, guión). Consideramos que el hecho de no haber incluido estos contenidos también en las Metas (2012) podría estar en el origen de los malos resultados expuestos en los informes de evaluación (ver más arriba).

En estos cuadros no puede apreciarse ninguna referencia al Diccionario Terminológico (2008), uno de los documentos que sustenta la terminología lingüística de uso obligatorio en educación. Por otro lado las Metas, en su introducción, incluyen la siguiente reflexión:

En muchos casos, los objetivos y respectivos descriptores fueron retomados ipsis verbis en la elaboración de las Metas Curriculares, otros fueron objeto de especificación, otros, sin embargo, fueron considerados como no integrantes de los contenidos fundamentales, que el presente documento define. (Metas, 2012, p.4) 
No es fácil, entonces, contestar a la pregunta ¿Cómo trabajar, a la par, con estos dos documentos? En primer lugar se confirma que las Metas (2012) constituyen una reducción de los Programas (2009) (cf. Teixeira, Correia y Pereira, 2014). Asimismo las Metas (2012) parecen constituirse como programas paralelos a los Programas (2009) en la medida en que también se presenta como un documento que define los "contenidos fundamentales" (p.4). No son claros los estatutos de ambos documentos: ¿Constituyen las Metas (2012) un programa para la asignatura de lengua? ¿Son otro programa? ¿Se solapan parcialmente a los Programas (2009)?

En rigor, es difícil contestar a estas preguntas porque en las Metas (2012) podemos leer, incluso, que el documento se presenta como "una nueva forma de organización de algunos contenidos" (p.5) y que responde a "la preocupación de formularlas [las Metas, 2012] de forma clara y precisa de tal forma que el profesorado sepa con exactitud $d^{i i i}$ lo que se pretende que el alumnado aprenda" (Metas, 2012, p. 5). De aquí se concluye que no siempre las intenciones de los legisladores se expresan adecuadamente en los textos normativos producidos.

\section{La relación Gramática-Escritura en los Programas (2009) y en las Metas (2012)}

Según lo definido en los Programas de Portugués para la Enseñanza Básica (Programa, 2009), deberá intensificarse la relación entre escritura y gramática. La escritura se define como "un proceso de fijación lingüístico que incluye el conocimiento del sistema de representación gráfica adoptado, así como los procesos cognitivos y translingüísticos complejos" (Programa, 2009, p. 16). Ello significa que deben materializar con corrección la aplicación de las reglas gramaticales establecidas para que un texto se pueda considerar correcto. Se considera que la reflexión explícita sobre la lengua tiene por objetivo una instrucción formal, intencionada, sobre la capacidad de "sistematizar unidades, reglas y procesos gramaticales del idioma, llevando a la identificación y a la corrección de las faltas" (Programa, 2009, p. 16), a la par de una reflexión permanente sobre la lengua.

Los procesos de análisis y reflexión se presentan, de hecho, como aspectos fundamentales para el desarrollo de la consciencia lingüística del alumnado. Ello posibilitaría transformar el conocimiento implícito sobre la lengua en conocimiento 
explícito. El aprendizaje de la gramática se presenta como factor de éxito escolar, por un lado, y como facilitador de calidad de vida de los futuros ciudadanos.

Parece, en este sentido, comprensible que desde muy temprano los resultados esperados al nivel de la escritura se relacionen estrechamente con los objetivos propuestos en el dominio del conocimiento explícito de la lengua (Programa, 2009). Los objetivos de la producción textual (por ejemplo, en relación a la aplicación de la norma ortográfica y las reglas de la puntuación) y de comprensión textual evidencian esta articulación. A nuestro entender esto tiene un impacto en la vida cotidiana de los alumnos. Una producción escrita u oral organizada, así como una correcta aplicación de las reglas de la puntuación, el uso adecuado del léxico y un buen dominio de la sintaxis contribuyen al conocimiento y dominio que el ciudadano tiene de su lengua, estando capacitado para utilizarla de la forma que mejor le convenga en función de sus objetivos comunicativos, profesionales, afectivos, etc.

En las Metas (2012), como ya hemos indicado, el conocimiento explícito de la lengua aparece con la designación de Gramática. Este documento intenta relacionar gramática y escritura en unos términos parecidos a los Programas. Así, afirma la necesidad de que

el alumnado adquiera y desarrolle la capacidad para sistematizar unidades, reglas y procedimientos gramaticales de la Lengua, de tal forma que pueda hacer uso sustentado del portugués en las diferentes situaciones de la oralidad, la lectura y de la escritura. (Programa, 2009, p. 6)

Queda, aún, por explicar el hecho de si la utilización en las Metas (2012) del término "Gramática" en lugar de "Conocimiento Explícito de la Lengua" (CLE en los Programas, 2009), no impide un trabajo basado en la enseñanza explícita de la gramática. Cualquier hablante posee, en el inicio del aprendizaje formal de la lengua y en grados diversos, un conocimiento de la gramática de su lengua materna (fonético, fonológico, morfológico, sintáctico, lexical, semántico y pragmático (Costa, Cabral, Santiago \& Viegas, 2011). Lo que el individuo necesita es reflexionar sobre sus características formales, mirándola desde fuera, como si de un objeto se tratase, demostrando habilidad para conocer y explicitar reglas y estructuras en diferentes situaciones específicas de uso. Por otra parte, es en este aspecto que se comprende la relación dialéctica entre la lingüística y la didáctica, en la medida que se profundiza en el conocimiento gramatical a través del conocimiento explícito del sistema de la 
lengua (cf. Viegas, 2013). Casteleiro (1989) alerta en este sentido de las implicaciones del desconocimiento de esta relación cuando afirma lo siguiente:

No ensino da língua portuguesa, a Linguística tem sido largamente utilizada como mero formalismo que em quase nada satisfaz aos objetivos da disciplina de Língua Portuguesa. Ou a Linguística contribui para a consecução de tais objetivos ou então ela revelar-se-á inútil como suporte da aula de português. (Casteleiro, 1989, p.61)

\section{Hacia una nueva metodología en la enseñanza de la gramática y de la escritura}

Después del análisis de los documentos normativos que rigen la enseñanza del portugués en relación al vínculo gramática-escritura, fácilmente se percibe que en ambos documentos se reconoce la importancia del conocimiento lingüístico para un adecuado desarrollo de la escritura. Los resultados obtenidos por el alumnado en los exámenes nacionales muestren que las dificultades evidenciadas en expresión escrita son, sobretodo, de índole gramatical. ¿Utilizará el profesorado en su práctica docente los Programas (2009) y las Metas (2012)? ¿Serán estos textos suficientemente explícitos en lo que se refiere al trabajo a desarrollar en estas áreas curriculares?

Creemos que estos documentos tienen algunos problemas a la hora de guiar a los profesores. Observaremos seguidamente el enfoque adoptado sobre los signos de puntuación, específicamente sobre el uso de la coma. Exploraremos el primer ciclo de la enseñanza básica (cursos de primero a cuarto) y el segundo ciclo (cursos quinto y sexto).

En relación a la Expresión Escrita. los Programas (2009) incluyen para los cursos primero y segundo el descriptor "Aplicar reglas de utilización de los signos de puntuación" y el contenido "Signos de puntuación: coma..." (p.41); para tercer y cuarto cursos el descriptor "Escribir textos (...) respetando la norma (orto)gráfica y las reglas de puntuación" (p.44); y para los cursos de quinto y sexto el descriptor "Respectar las reglas del uso de la puntuación" (p. 89).

En relación al Conocimiento Explícito de la Lengua podemos leer para los cursos primero y segundo el descriptor "Explicitar las reglas de la puntuación" así como el contenido "Signos de puntuación (...) coma (...) Uso de la como en contexto de enumeraciones" (p.53); para los cursos tercero y cuarto el descriptor "Explicitar reglas de la puntuación" y el contenido "Signos de puntuación (...) coma (prohibición de uso entre sujeto y predicado)" (p.60); y para los cursos quinto y sexto el descriptor 
"Explicitar las reglas de uso de los signos de puntuación para delimitar los constituyentes de la frase" (p. 99).

En los cursos primero y segundo se considera relevante, solamente, que el alumnado aprenda que la coma se utiliza en situaciones de enumeración, pero no que tenga la obligación de apropiarse del concepto en si mismo. En los cursos tercero y cuarto aparece la formulación de una regla que es considerada fundamental: prohibición del uso entre sujeto y predicado. Se expresa en la escritura a través de otra indicación que es el respeto por las convenciones de puntuación. Por lo que respecta a los cursos quinto y sexto, consideramos que se lograrían directrices más precisas explicitando las reglas de uso que los alumnos deben aprender. El uso de la coma no se confina a los dos casos estudiados (unos contextos supuestamente trabajados en el ciclo anterior).

Si el profesorado considera que las Metas (2012) funcionan como un programa de la asignatura, las indicaciones relativas al uso de la coma constituyen una “configuración" diferente. Se considera por ejemplo que la prohibición de uso de la coma entre el sujeto y el predicado no es un conocimiento prioritario, para el alumnado. Además, no se hace ninguna referencia a esta regla en las Metas (2012), en el dominio de la Gramática. Debemos subrayar que para el dominio de la Gramática no se incluye en las Metas (2012) ningún descriptor de nivel ni objetivo alguno relativo a la puntuación para los cursos primero y segundo. Sin embargo, para el dominio de la Lectura y de la Escritura se pretende que el alumnado identifique y utilice adecuadamente los signos de puntuación, como el punto (seguido, aparte y final), el punto de interrogación (en primer curso, Metas, 2012 p.10), las comas en situaciones de enumeración y coordinación de oraciones (en segundo curso, Metas, 2012, p.17) y las comas en contexto de movilidad de los elementos de la oración (en cuarto curso, Metas, 2012, p.30).

Nos preguntamos cómo consigue el alumnado usar en la escritura lo que no se ha trabajado en el dominio de la gramática. Específicamente nos formulamos la siguiente pregunta: ¿Es posible que el alumnado se exprese correctamente por escrito basándose en un conocimiento gramatical implícito, esto es, sin trabajar ese conocimiento de modo explícito? Creemos que no es posible. Así, defendemos que es de vital importancia que las tareas de gramática impliquen reflexión a través de procesos didácticos. Ello permitirá establecer puentes entre el conocimiento implícito 
de la gramática y el correspondiente conocimiento explícito, contribuyendo de esta forma a un adecuado nivel de expresión escrita. Presentamos a continuación dos actividades que pueden ser un modelo de intervención en el aula: el laboratorio gramatical y el juego didáctico. Creemos que este tipo de recursos tiene un gran potencial didáctico para el desarrollo de los aprendizajes relativos al uso de los signos de puntuación, en la escritura, y en especial para el uso (o prohibición de uso) de la coma.

\section{Laboratorio gramatical}

Consideramos que el recurso llamado Laboratorio Gramatical, por su carácter práctico y experimental, es positivo para el alumnado, en la medida en que de acuerdo con los resultados de investigación en diferentes países evidencian que plantea 'ventajas cognitivas'. Nosotros añadimos que puede ejercer también un impacto positivo en la expresión escrita (Teixeira, Gorgulho y Santos, 2014). De acuerdo con Duarte (2008), el uso del laboratorio gramatical no rechaza la “...necesidad de memorización de (...) reglas...", pero subraya que es necesario un seguimiento riguroso por parte del profesorado. La construcción de un laboratorio gramatical debe incluir las siguientes etapas: a) planificar (selección y preparación de datos), b) observar y describir datos, c) sistematizar los datos, y d) evaluar los resultados (Costa et al., 2011). Esta metodología parte del supuesto que el desarrollo del conocimiento gramatical es ventajoso para el alumnado. Se constituye como un reto, alejándose de ejercicios taxonómicos que pueden llevar al aburrimiento. ${ }^{\text {iv }}$ Debe estar formado por diferentes actividades dirigidas a los diferentes aprendizajes gramaticales, desarrolladas y recuperadas a lo largo del tiempo, sin que sean realizadas "de una sola vez" (Duarte, 2008, p. 19).

Se pretende que el laboratorio propuesto, previsto para segundo curso, sirva para explicitar una regla del uso de la coma prevista en los Programas (PPEB, 2009): prohibición de uso de la coma entre el sujeto y el predicado. Este contenido no aparece sin embargo en las Metas (2012) para ninguno de los cursos del primer ciclo de enseñanza básica. La razón que nos lleva a proponer el trabajo con esta regla se relaciona con la necesidad de que el alumnado escriba desde muy temprano (sea en la forma de frases sea en la forma de texto), aplicando las reglas de uso de los signos de 
puntuación (Programa, 2009). Proponemos trabajar esta regla en segundo porque pensamos que en primero podría ser prematuro.

Ejemplificamos esta actividad con la siguiente propuesta (desarrollada por Teixeira, et al., 2012). Presentamos un guión de orientación (dirigido al profesorado, Cuadro 3), en el cual se presenta lo estipulado en el marco curricular oficial: descriptores de nivel y contenidos de los Programas (2009). Nos basamos también en el Diccionario Terminológico (DT) (2008) y la Nova Gramática do Português Contemporâneo (Cunha y Cintra, 1999).

Cuadro 3. Guión

\begin{tabular}{|l|l|}
\hline Presentación & $\begin{array}{l}\text { Con esta tarea pretendemos trabajar desde el punto de vista didáctico el } \\
\text { uso de la coma, en el ámbito de la representación gráfica, en cuanto signo } \\
\text { de puntuación. El principal objetivo es conducir el alumnado a } \\
\text { situaciones de prohibición de uso de la coma entre el sujeto y el } \\
\text { predicado. }\end{array}$ \\
\hline Coma & $\begin{array}{l}\text { La coma marca en ocasiones una pausa de corta duración. Se usa no solo } \\
\text { para separar elementos en la oración, pero también en oraciones simples. } \\
\text { Los términos esenciales e integrantes de la oración se unen unos con otros } \\
\text { sin pausa; no pueden, de esta forma, ser separados con coma..." (Cunha y } \\
\text { Cintra, 1999, p.644). }\end{array}$ \\
\hline $\begin{array}{l}\text { Descriptores de nivel: } \\
\mathbf{2}^{\mathbf{0}} \text { curso de primaria. }\end{array}$ & $\begin{array}{l}\text { Manipular palabras (o grupos de palabras) en frases; comparar datos y } \\
\text { descubrir regularidades (procesos de concordancia); explicitar reglas y } \\
\text { procedimientos de puntuación (coma); movilizar el saber adquirido en la } \\
\text { lectura y en la escritura de palabras, frases y textos. (Programas, 2009, } \\
\text { p.53). }\end{array}$ \\
\hline $\begin{array}{l}\text { ¿Podemos usar la coma entre el sujeto y el predicado? } \\
\text { contesta }\end{array}$ & \begin{tabular}{l}
90 minutos. \\
\hline Tiempo previsto
\end{tabular} \\
\hline
\end{tabular}

El uso de las comas debe trabajarse de forma contextualizada a partir de textos concretos. Es necesario que el profesor muestre que en relación a las comas las normas de los discursos orales no tienen relación directa con las normas de los discursos escritos. Es importante que el alumnado entrene la memoria visual pero que también esté acompañado por el profesor en el proceso de fijar la atención en las regularidades, en los caos de prohibición de uso de las comas, pudiendo incluso "practicar el uso de regla" (Duarte, 2008, p. 19). Cabe destacar la importancia de formular interrogantes abiertos al final de la tarea. Estos interrogantes deben desencadenar la reflexión, posibilitando de esta forma caminos que lleven a descubrir la regla objeto de estudio. 


\section{El juego}

De acuerdo con Wittgenstein (1974), podemos asociar las reglas de la gramática a las reglas de un juego porque el estudio de la lengua presupone el conocimiento y el uso de las reglas. Según este autor podemos adoptar como base el juego de ajedrez y la forma como se mueven las piezas, en que es necesario seguir determinadas reglas fijas como en la gramática. Viegas (2013) presenta una reflexión basada en la lectura de la obra de Hjelmslev (1971), y concluye lo siguiente:

O jogo está para a língua, assim como as regras do jogo estão para as regras da gramática (...) a cada jogada poderá corresponder um texto. Assim como no jogo de xadrez onde com um número finito de peças e regras é possível realizar um número infinito de jogadas, também na língua é possível produzir um número infinito de textos com um número finito de unidades lingüísticas e de regras gramaticais. (Viegas, 2013, p.454)

A pesar de que desde un punto de vista lingüístico no compartimos esta perspectiva (muy cercana a los planteamientos chomskianos), consideramos que el conocimiento de la estructura de la lengua es importante en el campo de la didáctica. Así, el juego que proponemos presenta como objetivo evaluar el conocimiento y la aplicación de reglas gramaticales relativas al uso de las comas en quinto curso de primaria.

La selección de los contenidos relacionados con el uso de la coma se basa en el hecho de que el alumnado escribe textos, sea para aprender (aprender a escribir, a construir y a expresar conocimiento) sea para escribir en términos personales y creativos (Programa, 2009). Podemos ayudar así al alumno a sistematizar el conocimiento relacionado con el uso de las reglas de la lengua (en este caso del uso de la coma). Proponer esta actividad para quinto curso responde a la expectativa para el ciclo superior de que los alumnos escriban con corrección, respetando las reglas de la puntuación. Tal como podemos leer en las Metas (2012), los alumno deben poder aplicar "las reglas de uso de los signos de puntuación para representar tipos de frase y movimientos sintácticos básicos (enumerar, delimitar el vocativo, introducir grupos, separar de oraciones)" (p.38).

Proponemos una situación lúdica a través de una adaptación del Juego de la Oca y del Trivial Pursuit, que presenta dos situaciones de prohibición del uso de las comas: entre el sujeto y el predicado y entre el verbo y sus complementos (directo, indirecto y circunstancial). El juego se organiza a través de ítems de elección múltiple, de verdadero y falso y de preguntas de respuesta abierta (Cuadro 4). 
El ejemplo (titulado Entre Comas, y desarrollado en Teixeira, et al., 2012) se acompaña de un guión para el profesorado (Cuadro 5), que incluye descriptores de nivel y contenidos de acuerdo con los Programas (2009). Se presentan, igualmente, la fundamentación lingüística a partir del Diccionario Terminológico (DT) (2008) y la Nova Gramática do Português Contemporâneo (Cunha y Cintra, 1999).

Cuadro 4. Juego
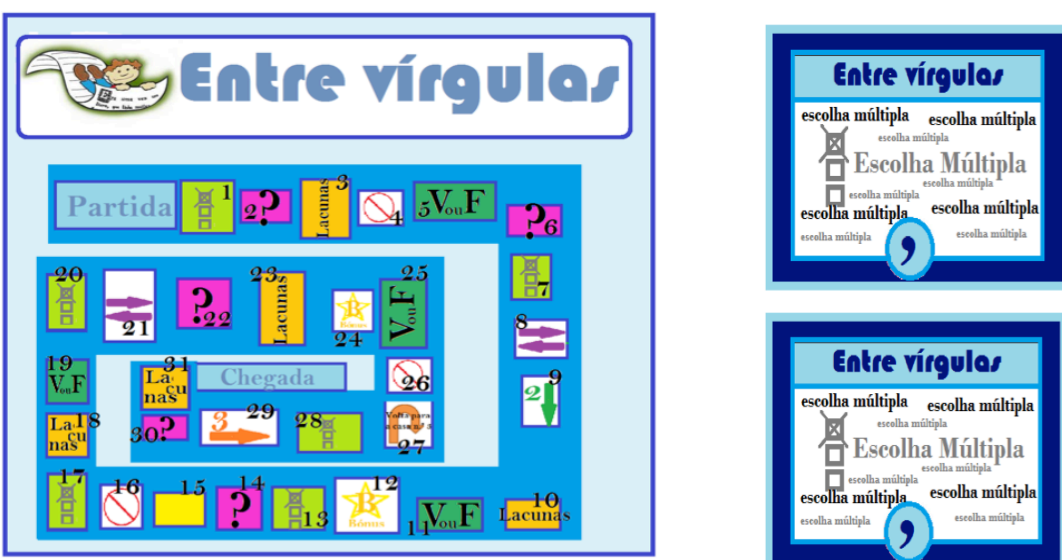

Qual das frases se encontra gramaticalmente correta?

a) $\mathrm{O}$ camião, explodiu aqui. b) 0 , camião explodiu aqui. c) 0 camião explodiu, aqui.

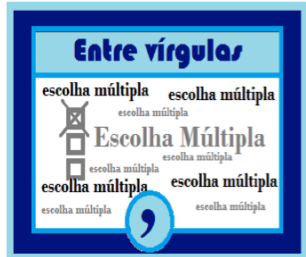

R.: alinea c)

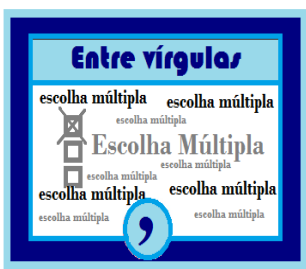

Qual das frases se encontra gramaticalmente correta?

a) A Renata, corre muito. b) A Renata corre muito. c) A Renata corre, muito.

Cuadro 5. Guión

\begin{tabular}{|l|l|}
\hline Presentación & $\begin{array}{l}\text { Con esta actividad se pretende desarrollar de forma lúdica el uso de las } \\
\text { comas, en el ámbito de la representación gráfica, en cuanto signo de } \\
\text { puntuación. El principal objetivo es conducir el alumnado a situaciones de } \\
\text { prohibición de uso de las comas entre sujeto y predicado y en el interior de } \\
\text { éste entre predicado y complemento directo. }\end{array}$ \\
\hline Uso de las comas & $\begin{array}{l}\text { Las comas marcan en ocasiones una pausa de corta duración. Se utiliza no } \\
\text { sólo para separar elementos en una oración sino también para separar } \\
\text { oraciones simples. Los términos esenciales e integrantes de la oración se } \\
\text { unen sin pausa; no puede, así, ser separados por comas (Cunha y Cintra, } \\
1999, \text { p.644) }\end{array}$ \\
\hline $\begin{array}{l}\text { Descriptores de nivel: } \\
\text { 5 curso de primaria }\end{array}$ & $\begin{array}{l}\text { Explicitar reglas de uso de los signos de puntuación (...) (Programas, 2009, } \\
\text { p.99) }\end{array}$ \\
\hline $\begin{array}{l}\text { Preguntas a las que } \\
\text { contesta }\end{array}$ & ¿Cuándo debemos usar las comas y cuiándo no debemos usarlas? \\
\hline Tiempo Previsto & 90 minutos. \\
\hline
\end{tabular}


El objetivo del juego es promover el conocimiento del uso de las comas entre los alumnos. Respetando el principio de la progresión, las preguntas se hacen siguiendo una progresión creciente de dificultad. Optamos por diferentes tipologías de pregunta y respuesta para proporcionar al alumnado distintas formas de razonar y de expresar su pensamiento. Así, una determinada situación se evaluará como correcta o incorrecta (en las respuestas de verdadero y falso) mientras que en otros casos (selección de respuesta correcta de entre diferentes opciones en las preguntas de elección múltiple) se promueve el contraste entre posibles soluciones; finalmente las tareas de completar textos promueven la reflexión sobre los principios del sistema.

\section{Consideraciones finales}

Los resultados de los exámenes nacionales constituyen una prueba de los bajos conocimientos del alumnado sobre gramática y escritura. Sugieren que la falta de conocimiento gramatical tiene implicaciones en el nivel de expresión escrita, ya sea en términos morfológicos, ya sea en términos sintácticos, interfiriendo también en los mecanismos de cohesión y coherencia textual.

En este texto hemos explorado si estos resultados podían ponerse en relación a los documentos normativos que regulan la práctica pedagógica: los Programas del área curricular de Portugués (1er y $2^{\circ}$ ciclos de la Enseñanza Básica) y la asignatura de Portugués (3er ciclo de la enseñanza Básica) (2009) (a los que nos hemos referido con el término Programas, 2009) y las Metas Curriculares de Portugués para la Enseñanza Básica (2012) (a las que nos hemos referido con el término Metas, 2012).

Hemos observado que estos documentos se organizan de forma diferente. Por un lado los Programas (2009) están organizados en función de los diferentes ciclos de la enseñanza básica (primer, segundo y tercer ciclo) y por competencias específicas (Comprensión Oral, Expresión Oral, Lectura, Escritura, Conocimiento Explícito de la Lengua). Por otro lado las Metas (2012) se organizan por curso (cursos $1^{\circ}, 2^{\circ}, 3^{\circ}, 4^{\circ}$, $5^{\mathrm{o}}$ y $6^{\circ}$ ) y dominios (Oralidad, Lectura y Escritura, Educación Literaria y Gramática). Hemos remarcado que por ello podríamos considerar las Metas (2012) como un programa de asignatura pues presentan una selección de los contenidos de los Programas (2009) considerados como prioritarios.

Hemos observado que en ambos documentos se considera que un buen conocimiento gramatical conlleva un buen nivel de expresión escrita. A pesar de ello 
hemos visto que hay diferencias entre ellos: los descriptores de nivel y los contenidos respectivos no siempre tienen una correspondencia clara. Este hecho no parece que contribuya esclarecer la tarea del profesorado.

Hemos presentado dos propuestas didácticas cuyo objetivo es el trabajo del uso (o prohibición de uso) de las comas, con ejemplos de orientación metodológica, una para segundo curso de primaria (Laboratorio gramatical) y otra para quinto curso (Juego). No se trata en ninguno de los dos casos de recetas para el éxito del alumnado: es preciso, por ello, que el docente explore la tipología de los contenidos que los alumnos deberán aprender, así como considerar el contexto del grupo. Además, creemos que será siempre útil y productivo que el profesorado proporcione momentos de aprendizaje que varíen entre lo lúdico y la enseñanza explícita de los contenidos, porque diferentes alumnos adquieren los conocimientos de diferentes maneras y a través de diferentes tipologías de estrategia, según su tipo de inteligencia individual.

La temática que desarrollamos en esta propuesta no se agota en el análisis de los programas de la asignatura de portugués y en propuestas didácticas. Los libros de texto son otro aspecto que merece también la atención de la investigación. Estos materiales a veces se alejan de las orientaciones consignadas en los programas. A menudo sus autores optan por los Programas (2009) (cf. Teixeira, Lopes y Gorgulho, 2014). Podríamos inferir que si los libros de texto están de acuerdo con los Programas (2009), serían coherentes también con las Metas (2012), pues en este último documento se indica lo que es prioritario en aquél. Sin embargo esta idea no se ve siempre reflejada en los materiales. Además, el Ministerio de Educación ha impuesto recientemente que los libros de texto ya evaluados y aprobados de acuerdo con los Programas (2009) sean de nuevo evaluados de acuerdo con las Metas (2012). Desde nuestro punto de vista esto sugiere la existencia de posibles divergencias en algunos libros en relación a ambos documentos y los problemas que ello puede entrañar para la práctica docente.

Otro aspecto que nos merece una atención particular es la formación del profesorado, tanto en formación inicial como en formación continua. Debe "tener bases científicas sólidas y apoyarse en las didácticas y en el conocimiento sobre la práctica docente" (Diário da República, 2008). El profesor debe ser, cada vez más, un investigador permanente que puede ir más allá de los conocimientos científicos y didácticos desarrollados en la formación inicial. Debe implicarse en una búsqueda 
permanente y crítica de respuestas que sirvan para dar respuestas variadas a diversidad de la realidad educativa. Ello le puede ayudar a contribuir con ideas innovadoras al desarrollo de competencia gramatical de los alumnos, más allá de los libros de texto entendidos como guías legitimadores de las metodologías de enseñanza y de aprendizaje.

Defendemos vivamente la necesidad en Portugal de una mayor articulación y conformidad entre los documentos oficiales que rigen la práctica docente; una mayor apuesta por la diversidad de metodologías utilizadas en el aula; y una mayor inversión en la formación del profesorado, como actores críticos en escenarios cambio. Nos parece necesario que el Ministerio de Educación dé una mayor atención a todo un conjunto de factores externos e internos al sistema que pueden interferir negativamente en los procesos de enseñanza y aprendizaje, en este caso de la gramática y la escritura.

\section{Referencias bibliográficas}

Casteleiro, J.M. (1989). Formar professores de língua Portuguesa: com que linguística? En Actas do Congresso de Sobre a Investigação e o Ensino do Português. Lisboa: ICLP.

Costa, J., Cabral, A., Santiago, A., Viegas, F. (2011). Guião de implementação do programa de PortuguÊS do ensino básico - conhecimento explícito da ingua. Lisboa: Ministério da Educação - DGIDC.

Cunha, C., \& Cintra, L. (1999). Nova gramática do Português contemporâneo. Rio de Janeiro: Nova Fronteira.

Diário da República, $2^{\mathrm{a}}$ série - N²28 - 24 de Novembro de 2008. Parecer n ${ }^{\circ} 8 / 2008$.

Duarte, I. (2008). O conhecimento da língua: Desenvolver a consciência linguística. Lisboa: Ministério da Educação.

Guião de Implementação do programa de português para o ensino básico conhecimento explícito da língua. (2011). Lisboa: Ministério da Educação DGIDC

GAVE-Gabinete de Avaliação da Educação (2010) Relatório do Exame Nacional de 2010. Lisboa: Ministério da Educação. http://www.gave.minedu.pt/np3content/?newsId=24\&fileName=Exames_2010_Fase1_Ch1.pdf

GAVE-Gabinete de Avaliação da Educação (2011) Relatório do Exame Nacional de 2011. Lisboa: Ministério da Educação. http://www.gave.minedu.pt/np3content/?newsId=24\&fileName=Rel_Exames_2011.pdf

GAVE-Gabinete de Avaliação da Educação (2012) Relatório do Exame Nacional de 2012. Lisboa: Ministério da Educação. http://www.gave.minedu.pt/np3content/?newsId=24\&fileName=RelExames_2012_23jul.pdf.

GAVE- Gabinete de Avaliação da Educação (2013) Projeto Testes Intermédios. $1^{o}$ Ciclo do Ensino Básico 2013. Lisboa: Ministério da Educação. http://www.gave.min- 
edu.pt/np3content $/$ ?newsId $=24 \&$ fileName=PrelimReport_Exams_2013_PDFCo n.pdf

Hjelmslev, L. (1971). Prolegómenos a una teoría del lenguaje. Madrid: Gredos.

MCPEB (2012). Metas Curriculares de Português para o Ensino Básico. Lisboa: Ministério da http://dge.mec.pt/metascurriculares/index.php?s=directorio\&pid=16

PPEB (2009). Programa de Português para o Ensino Básico. Lisboa: Ministério da Educação.

Sá, C. (2012). Transversalidades da língua portuguesa: representações, instrumentos e formação. Revista Exedra, 364-372.

Scriven, M. (1967). The methodology of evaluation. En R. M. Tyler (org.) Perspectives of curriculum evaluation (pp. 39-83). Chicago: Rand McNally.

Teixeira, M.; Correia, R. \& Pereira, S. (2014). Avaliar para conhecer. A avaliação externa vs. documentos norteadores da Prática letiva (des) articualção? En C. Tomás, \& C. Gonçalves (orgs.). Atas do VI encontro do CIEB - I encontro internacional em estudos educacionais. Avaliação: Desafios e riscos (pp. 434450) Lisboa: Centro Interdisciplinar de Estudos Educacionais.

Teixeira, M., Gorgulho, A. R., \& Santos, S. (2014). Gramática e escrita - que relação?" Comunicação oral, no I congreso internacional sobre la enseñanza de la gramática - presente, passat i futur. Universidade de Valência, Valência, 28 de fevereiro.

Teixeira, M., Lopes, S., \& Gorgulho, A. R. (2014). Gramática e manuais escolares que avaliação? Comunicação oral I congreso internacional sobre la enseñanza de la gramática - presente, passat i futur. Universidade de Valência, Valência, 28 de fevereiro.

Teixeira, M., Soares, I., Reis, F., Correia, R., Carvalho, B., \& Galrinho, J. (2012). O uso da vírgula e as funções sintáticas - propostas de abordagem" VI encuentro de la lengua portuguesa en los $1^{\circ} \mathrm{s}$ cursos de la escolaridad obligatoria Investigación y buenas prácticas, en la escola superior de educação de lisboa. VI encuentro de la lengua portuguesa en los $1^{\circ} \mathrm{s}$ cursos de la escolaridad obligatoria - Investigación y buenas prácticas, en la Escola Superior de Educação de Lisboa: Lisboa, 9 de julio.

Travaglia, L.C. (2007). A gramática na escola / língua Portuguesa: o ensino de gramática. Salto para o futuro. Boletim, 3, 73 - 97.

Viegas, F. (2013). A gramática: um jogo no ensino e na aprendizagem do português. En Madalena Teixeira et al. (orgs.) Ensinar e aprender português num mundo plural (pp. 450-465). Santarém: Escola Superior de Educação de Santarém e Universidade Federal de Uberlândia.

Wittgenstein, L. (1974). Philosophical grammar. Oxford: Blackwell.

\footnotetext{
i Los trabajos desarrollados para la elaboración de este texto han contado con la colaboración de Ana Rita Gorgulho y Sandra Lopes. Debemos un agradecimiento especial a Sílvia Melo-Pfeifer por sus comentarios y a António Pais por una primera traducción del texto al castellano; todas las traducciones de las citas son de nuestra responsabilidad. Agradecemos a Xavier Fontich la presente versión final en castellano, fruto de una reducción del texto original así como de una revisión normativa, estilística y tipográfica. Agradecemos muchísimo, aún, al Xavier Fontich por la invitación de poder colaborar en este prestigiado journal.

ii En adelante, usaremos los términos Metas -como substitución de MCPEB- y Programas -como substitución de PPEB-, para facilitar la comprensión del texto y de los documentos de referencia.
} 
iii El subrayado es nuestro.

iv "Me aburro" o "¿Para qué sirve esto?" son algunas de las opiniones de alumnos de $4^{\circ}$ curso de primaria en el 2014, en un colegio público de la ciudad de Santarém, recogidas en el ámbito de la supervisión de las prácticas de primaria. Tarea incluida en el Prácticum de un estudiante (Universidad de Lisboa).

Referencia de la autora:

Madalena Teixeira es professora adjunta a la Universidade de Lisboa. Ella es investigadora al Centro de Estudos Anglísticos (CEAUL).

Email: madalena.dt@gmail.com

Para citar este artículo:

Teixeira, M. (2014). Relación entre gramática y escritura en el currículo de lengua Portuguesa. Bellaterra Journal of Teaching \& Learning Language \& Literature, 7(2), 1-20. 